\title{
SHARING ECONOMY E DIRITTO DI ACCESSO A INTERNET: LE SFIDE DEL DIGITALE NEL COSTITUZIONALISMO MULTILIVELLO ${ }^{1}$
}

SHARING ECONOMY AND RIGHT TO INTERNET ACCESS: THE CHALLENGES OF THE DIGITAL TRANSFORMATION IN THE MULTILEVEL CONSTITUTIONALISM

Roberto Miccú

Giacomo Roma ${ }^{2}$

\section{SOMMARIO}

Nella società della rivoluzione digitale, essere connessi a Internet appare come un'esigenza fondamentale. In diversi ordinamenti si è posta la questione del riconoscimento di tale diritto, che in alcuni casi è giunta fino alla sua affermazione a livello costituzionale. La necessità di garantire una connessione a Internet veloce, affidabile e sicura è ancor più sentita nel contesto di rapida evoluzione che alcuni servizi hanno conosciuto negli ultimi anni, nella transizione verso la sharing economy.

Descrittori: Sharing economy. Economia della condivisione, Diritto di accesso a Internet. Costituzionalismo multilivello. Diritti Fondamentali. Servizio universale. Uber.

\section{ABSTRACT}

In the digital revolution, Internet connection seems to be a fundamental need. Different legal orders have faced the issues of the recognition of such right, which in some cases has been stated at constitutional level. A fast, reliable and 
secure Internet connection is much needed in the context of swift development of some services in the last years, in the transition towards the sharing economy.

Keywords: Sharing economy. Right to Internetaccess. Multilevel constitutionalism. Fundamental Rights. Universal servisse. Uber.

\section{INTRODUZIONE}

Nella società della rivoluzione digitale, essere connessi a Internet appare come un'esigenza fondamentale: dall'acquisizione di informazioni e conoscenze, all' accesso a beni e servizi, fino all'esercizio di alcuni diritti di cittadinanza, la rete rappresenta uno strumento ormai indispensabile. Essere esclusi dal suo utilizzo può determinare situazioni di marginalità simili a quelle legate, in passato, all'impossibilità di fruire di determinate prestazioni. Si pensi ad esempio al servizio postale, o ai trasporti: là dove fino a epoche recenti si riteneva indispensabile consentire a ciascuno il diritto di spostarsi e necessario fornire i mezzi per soddisfare questo bisogno, oggi quest'esigenza può essere considerata sostituibile con quella dell'accesso a una connessione internet, che evita la necessità di muoversi fisicamente, consentendo tuttavia lo svolgimento della medesima attività.

Tuttavia, a livello globale, si registrano ancora forti squilibri nell'accesso a Internet e in particolare a Internet veloce, la cosiddetta banda larga, che dà il nome a quello che viene definito come un right to braodband ${ }^{3}$. La Banca Mondiale, che definisce l'accesso a Internet veloce come una "necessità fondamentale per lo sviluppo economico e umano sia nei Paesi sviluppati che in quelli in via di sviluppo", ricorda come nelle economie avanzate sia circa l' 80 per cento della popolazione ad avere tale accesso, contro il 35 per cento dei paesi in via di sviluppo. Portare questo livello al 75 per cento consentirebbe, secondo la Banca Mondiale, di aumentare il prodotto interno lordo di questi paesi di duemila miliardi di dollari e creare oltre 140 milioni di posti di lavoro. Nei paesi dell'OCSE, si registra una sostanziale corrispondenza tra numero di abitanti e abbonamenti a Internet ad alta velocità. Tuttavia, le divergenze in termini di velocità della connessione sono molte. Se si 
guarda all'Italia, si osserva un numero elevato di abbonamenti alla banda larga (87,6 ogni 100 abitanti, contro i 79,8 della Germania e i 76,5 della Francia), ma uno sviluppo ancora limitato della fibra ottica (circa il 3 per cento degli abbonamenti, contro il 21 per cento della media OCSE, anche se Germania e Francia sono su livelli comparabili).

La necessità di garantire una connessione a Internet veloce, affidabile, sicura è ancor più sentita nel contesto di rapida evoluzione che alcuni servizi hanno conosciuto negli ultimi anni, nella transizione verso la c.d. sharing economy o economia della condivisione ${ }^{4}$. Essa, infatti, si basa su piattaforme digitali che consentono di mettere in relazione tra loro utenti e fornitori di servizi. Innovando le modalità di fruizione di alcune prestazioni - si pensi, ad esempio, alla ricerca di un alloggio tramite Airbnb rispetto al tradizionale settore alberghiero -, il digitale richiede, quindi, un'evoluzione del quadro regolatorio che caratterizza alcuni comparti, oltre a mettere in discussione alcune categorie giuridiche apparentemente consolidate.

In questo scritto, dopo aver esaminato gli strumenti giuridici posti a garanzia del diritto di accesso a Internet, ci soffermeremo quindi sul portato innovativo della sharing economy, in particolare nell'ordinamento europeo multilivello.

\section{L'ACCESSO A INTERNET, UN DIRITTO FONDAMENTALE?}

In vari ordinamenti si è posta la questione della costituzionalizzazione del diritto di accesso a Internet e sulla natura di tale diritto: diritto sociale? Corollario della libertà di espressione? Diritto civile a sé stante ${ }^{5}$

Una posizione avanzata sul punto è stata adottata, sul piano giurisprudenziale, in Francia. Il giudice costituzionale, chiamato a giudicare la legittimità costituzionale di norme che sanzionavano con la sospensione dell'abbonamento il download illegale da Internet (c.d. legge $\mathrm{HADOPI}^{6}$ ), ha riconosciuto che "nello stato attuale dei mezzi di comunicazione e tenuto conto dello sviluppo generalizzato dei servizi di comunicazione al pubblico attraverso la rete nonché dell'importanza acquisita da questi per la partecipazione alla vita democratica e l'espressione 
di idee e opinioni, [la libertà di comunicazione delle idee e delle opinioni] implica la libertà di accedere a questi servizi"7. È significativo che questa decisione riconduca il diritto di accesso a internet nell'alveo dell'articolo 11 della Dichiarazione dei diritti dell'uomo e del cittadino, che riconosce la libera comunicazione delle idee e delle opinioni come "uno dei diritti più preziosi dell'uomo". Non viene però consacrato un vero e proprio "diritto di accesso alla rete", che avrebbe condotto a garantire a ognuno un diritto di carattere generale e assoluto. Ciò risente certamente della necessità di conciliare tale diritto individuale con il diritto di proprietà, cui vengono ricondotti i diritti di proprietà intellettuale. Ma testimonia anche una certa difficoltà nel riconoscere questo diritto in quanto tale, in particolare per le implicazioni in termini di predisposizione delle necessarie infrastrutture che ciò comporterebbe.

Nel diritto positivo, uno dei testi più avanzati in materia è il c.d. Marco Civil da Internet, approvato in Brasile con la legge n. 12.965 del 23 aprile 2014. Il testo fissa alcuni principi della disciplina dell'uso di internet, quali il rispetto della libertà di espressione, la protezione della privacy e dei dati personali, la neutralità della rete, la tutela di stabilità, sicurezza e buon funzionamento della rete, attraverso le misure tecniche previste dagli standard internazionali. Gli obiettivi perseguiti dalla normativa brasiliana sono il diritto di tutti ad accedere a Internet, il libero accesso all'informazione, alla conoscenza e alla partecipazione nella vita culturale e nella gestione della cosa pubblica, l'innovazione e lo stimolo alla più ampia diffusione delle nuove tecnologie e dei diversi modelli di utilizzo e accesso, l'adozione di standard tecnologici di tipo aperto, che consentono la comunicazione, l'accessibilità e l'interoperabilità tra applicazioni e banche dati diverse.

Il Marco Civil si configura come un vero e proprio Bill of Rights di Internet, elencando una serie di diritti e doveri degli utenti per l'accesso a Internet, qualificato come "essenziale per l'esercizio dei diritti di cittadinanza". I diritti riconosciuti dal Marco Civil possono essere ricompresi in diverse categorie.

La prima riguarda il rispetto della vita privata. Viene infatti citata l'inviolabilità della privacy, della segretezza dei flussi d'informazione trasmessi attraverso Internet e delle comunicazioni private conservate 
in rete (a eccezione di quanto ordinato da provvedimenti dell'autorità giudiziaria). Questi principi sono corredati da obblighi di trasparenza, che prevedono sia la completezza delle informazioni che il provider deve fornire quanto alle condizioni di conservazione dei tracciati di accesso a internet sia la necessità di raccogliere il consenso dell'utente, e dal divieto di diffusione presso terzi degli stessi.

In secondo luogo, vengono riconosciuti dei veri e propri "diritti alla connessione", quali il divieto di sospendere l'accesso a Internet, a eccezione dei casi di morosità derivanti direttamente dal suo utilizzo, e il mantenimento della qualità della connessione concordata con il provider.

Il Marco si sofferma, inoltre, sulla neutralità della rete, sancendo che il soggetto responsabile della trasmissione ha il dovere di processare qualsiasi flusso di dati, senza distinguere in base al contenuto, all'origine, alla destinazione, al tipo di servizio o applicazione - a eccezione delle esigenze tecniche essenziali o necessari per dare priorità ai servizi di emergenza.

Un capitolo del Marco è interamente dedicato al ruolo dei soggetti pubblici. Esso riguarda prevalentemente lo sviluppo della rete, la cittadinanza digitale e il miglioramento delle competenze digitali. Per quanto riguarda il primo aspetto, ai poteri pubblici è dato il compito di migliorare le infrastrutture di rete e promuovere lo sviluppo di data center, la qualità tecnica, l'innovazione e la diffusione delle applicazioni Internet, senza pregiudizio per il carattere aperto, neutrale e partecipativo della rete. In materia di cittadinanza digitale e e-government, risulta centrale garantire l'interoperabilità dei servizi, la diffusione delle informazioni in open data, l'adozione di tecnologie oper source. Il miglioramento delle competenze digitali deve invece tendere alla promozione dell'inclusione digitale, la riduzione delle differenze tra regioni del Paese e palla produzione e diffusione dei contenuti di origine nazionale.

In Italia, manca una consacrazione così completa del diritto di accesso a Internet, anche se il dibattitto sul riconoscimento del diritto di accesso a Internet si è acceso, nel corso della scorsa legislatura, intorno a delle proposte di revisione costituzionale, che però non sono giunte all'approvazione definitiva ${ }^{8}$. La questione resta, dunque, aperta. La proposta AS 1317 prevedeva di inserire all'articolo 21 della Costituzione 
il seguente comma: "Tutti hanno il diritto di accedere liberamente alla rete internet. La Repubblica rimuove gli ostacoli di ordine economico e sociale al fine di rendere effettivo questo diritto. La legge promuove e fornisce le condizioni per lo sviluppo della tecnologia informatica". Un'altra, che privilegiava l'ipotesi dell'inserimento di un articolo 34-bis nel titolo dedicato ai rapporti etico-sociali, era così formulata: "Tutti hanno uguale diritto di accedere alla rete Internet, in modo neutrale, in condizioni di parità e con modalità tecnologicamente adeguate. La Repubblica promuove le condizioni che rendono effettivo l'accesso alla rete Internet come luogo ove si svolge la personalità umana, si esercitano i diritti e si adempiono $i$ doveri di solidarietà politica, economica e sociale" (AC 2816 e AS 1561).

Nell'ordinamento dell'Unione europea, solo con il regolamento $2015 / 2120$ del 25 novembre $2015^{9}$ è stato riconosciuto il diritto degli utenti finali di "accedere a informazioni e contenuti e di diffonderli, nonché di utilizzare e fornire applicazioni e servizi, e utilizzare apparecchiature terminali di loro scelta, indipendentemente dalla sede dell'utente finale o del fornitore o dalla localizzazione, dall'origine o dalla destinazione delle informazioni, dei contenuti, delle applicazioni o del servizio, tramite il servizio di accesso a Internet".

La fornitura di tale servizio viene ricondotta alle categorie più tradizionali di "servizio di interesse economico generale" (SIEG) ${ }^{10}$ e "servizio universale"11.

La definizione di SIEG implica l'ampio potere discrezionale delle autorità nazionali di fornire servizi il più vicini possibile alle esigenze degli utenti, il rispetto della diversità tra i vari SIEG e tra le diverse esigenze degli utenti in ragione di situazioni geografiche, sociali e culturali diverse, nonché un alto livello di qualità, sicurezza e accessibilità economica, la parità di trattamento e la promozione dell'accesso universale e dei diritti dell'utente ${ }^{12}$. La qualificazione di un'attività come SIEG comporta la sottoposizione delle imprese incaricate della loro gestione alle regole di concorrenza, nei limiti in cui l'applicazione di tali norme non osti all'adempimento, in linea di diritto e di fatto, della specifica missione loro affidata (art. 106, par. 2 TFUE). Quando un'attività viene considerata un SIEG, ai fornitori vengono imposti degli obblighi di servizio pubblico, per cui essi devono ricevere adeguata compensazione. Gli OSP non possono 
invece essere attribuiti a un'attività che è già fornita o che può essere fornita in modo soddisfacente a condizioni compatibili con il pubblico interesse da imprese operanti in normali condizioni di mercato ${ }^{13}$.

La Commissione europea ha ricordato le condizioni a cui la nozione di SIEG può essere applicata a la creazione della rete Internet a banda larga. In particolare, "l'installazione e il funzionamento di un'infrastruttura di banda larga come SIEG possono essere ammessi solo se tale infrastruttura offre una connessione universale a tutti gli utenti di una regione determinata, tanto all'utenza residenziale quanto a quella commerciale"14. Ciò implica, inoltre, che il fornitore della rete da sviluppare non potrà rifiutare l'accesso all'ingrosso all'infrastruttura in base a criteri discrezionali e/o discriminatori. Tale qualificazione costituisce un requisito essenziale per l'intervento pubblico nella costruzione delle infrastrutture digitali.

Ma la categoria dei SIEG non è l'unica alla luce della quale deve essere letto l'accesso a Internet nel nostro ordinamento. Dal 2010, esso è stato inserito nella legislazione europea sul servizio universale delle telecomunicazioni. Il servizio universale è il principio secondo cui tutti i cittadini devono disporre di un'ampia gamma di servizi di base di buona qualità, a prezzi contenuti, in modo tale da partecipare pienamente alla vita sociale. La difficoltà nel disegnare il "servizio universale di accesso a Internet" risiede nella veloce mutazione tecnologica: velocità di connessione che, fino a tempi recenti, potevano essere considerate come un "servizio di base ma di buona qualità" appaiono oggi come obsolete. Inoltre, la fissazione di una velocità minima di connessione uguale in tutta l'Unione europea potrebbe distorcere e ridurre la concorrenza, limitando gli investimenti privati nei progetti di infrastruttura digitale.

La direttiva 2009/136/CE del 29 novembre 2009, che ha modificato la c.d. direttiva servizio universale (DSU) del $2002^{15}$, ha previsto che dovesse essere fornita da almeno un'impresa una connessione "in grado di supportare le comunicazioni vocali, facsimile e dati, a velocità di trasmissione tali da consentire un accesso efficace a Internet, tenendo conto delle tecnologie prevalenti usate dalla maggioranza degli abbonati e della fattibilità tecnologica" (nuovo art. 4, par. 2 della DSU).

Va notato come gli Stati membri dispongano di un'ampia discrezionalità nel determinare il contenuto del servizio universale: 
questo può, in particolare, significare che la velocità fornita è inferiore a quella dei servizi a banda larga o in fibra ottica. Secondo un recente censimento della rete europea dei regolatori delle telecomunicazioni (Body of European Regulators for Electronic Communications - BEREC), solo in sette paesi europei la banda larga è coperta dalla definizione di servizio universale. Interessante è lo studio approntato dal regolatore britannico Ofcom (Office of Communications) sull'introduzione del servizio universale per la banda larga nel Regno Unito, che ha diversi scenari. Il primo prevede un servizio standard corrispondente a una velocità di download di $10 \mathrm{Mbit} / \mathrm{s}$; nel secondo si aggiunge una velocità di upload di $1 \mathrm{Mbit} / \mathrm{s}$, un tempi di risposta (latency) medi, un livello massimo di condizione tra consumatori (50:1) e un limite allo scambio di dati basato sui profili di utilizzo più comuni (100 GB al mese); in quello più avanzato, la velocità di download viene portata a $30 \mathrm{Mbit} / \mathrm{s}$, quella di upload a $6 \mathrm{Mbit} / \mathrm{s}$, tempi di risposta rapidi, una banda minima garantita di 10Mbit/s e l'eliminazione dei limiti alle capacità di utilizzo.

La possibilità di disporre di tali velocità di connessione dovrebbe mettere tutti gli utenti in condizione di accedere a servizi forniti attraverso piattaforme digitali, che sono quelli che caratterizzano la sharing economy.

\section{LA SHARING ECONOMY NEL CONTESTO ITALIANO ED EUROPEO}

La categoria dei beni "in comune" (shared), che caratterizzano l'economia collaborativa, ha assunto una crescente rilevanza economica e giuridica ${ }^{16}$. Il fenomeno sottostante è quello della c.d. servificazione dell'economia, del passaggio dalla categoria della proprietà a quella dell'uso temporaneo. C'è chi attribuisce a questa trasformazione una forte connotazione di tipo idealistico, esaltando il lato solidaristico e sociale che la sharing economy certamente presenta e che ha contribuito alla sua affermazione e diffusione. Tale fenomeno ha potuto nascere e svilupparsi grazie alla diffusione di apparati tecnologici, e in particolare degli smartphone, che consentono a qualsiasi utente-consumatore, 
in ogni momento e contestualmente di stabilire la propria posizione, assumere informazioni sull'offerta di servizi a disposizione, concludere un contratto, effettuare una transazione finanziaria. La facilità di concludere un negozio giuridico che in altri tempi avrebbe richiesto diverse operazioni complesse, insieme a un livello di standardizzazione tecnologica che ha creato un mercato virtualmente globale, determina la possibilità di un utilizzo più efficiente di beni e servizi che eravamo abituati a concepire e utilizzare secondo dinamiche di proprietà esclusiva.

Non ci troviamo, quindi, di fronte a categorie giuridiche nuove. Gli appartamenti sono sempre stati dati in locazione, anche per breve periodo, le macchine sono sempre state noleggiate, i vicini si sono sempre prestati tra loro gli utensili per la cura del giardino, così come si è sempre trovato qualcuno disposto a fare piccoli lavori a casa, magari in cambio della promessa di aiuto in futuro. Ma le dimensioni che il fenomeno ha assunto grazie alla massificazione e standardizzazione dell'utilizzo di internet, insieme alla nascita e alla crescita, in certi casi esponenziale, di alcune piattaforme informatiche di intermediazione, ha fatto sì che le trasformazioni abbiano acquisito un carattere qualitativo e non più quantitativo. È accaduto, in particolare, che questi fenomeni economici abbiano inciso su mercati storicamente regolati - in certi casi, forse, ultra regolati - e il cui funzionamento faceva parte non solo del nostro sistema economico ma anche della nostra cultura. Il riferimento è, ad esempio, al mercato dei taxi, delle locazioni temporanee, delle prestazioni d'opera, ma i casi possono essere e sono molti di più.

Per gli studiosi di diritto dell'economia il fenomeno è affascinante: come reagisce la regolazione esistente rispetto a una modifica di massa delle dinamiche negoziali, proveniente sia dall'alto, da parte di società multinazionali, sia dal basso, da parte dell'insieme degli utenti che in ogni momento possono scaricare una applicazione e iniziare a utilizzarla? E in particolare, come reagiscono o stanno reagendo i regolatori e i soggetti che erano abituati a operare in mercati regolati quali imprenditori?

Nell'ordinamento europeo, il documento che assume maggiore rilevanza è la comunicazione della Commissione europea Un'agenda Europea per l'economia collaborativa, adottata a giugno del $2016^{17}$. La comunicazione, come spesso avviene con questo strumento di 
soft law, analizza il fenomeno della sharing economy, evidenzia le criticità in atto e potenziali, ipotizza possibili scenari di sviluppo delle politiche e della regolazione dell'Unione. Si riconosce, in particolare, che l'economia collaborativa "possa dare un contributo importante alla crescita e all'occupazione nell'Unione europea, se promossa e sviluppata in modo responsabile", evidenziando i vantaggi per i consumatori in termini di accesso a un numero più ampio di servizi e di aumento della loro qualità, nonché quelli per l'ambiente derivanti da un utilizzo più efficiente delle risorse.

Nella definizione che è stata accolta dalla Commissione, l'economia collaborativa comprende tre categorie di soggetti: "i) i prestatori di servizi che condividono beni, risorse, tempo e/o competenze e possono essere sia privati che offrono servizi su base occasionale ("pari") sia prestatori di servizi nell'ambito della loro capacità professionale ("prestatori di servizi professionali"); ii) gli utenti di tali servizi; e iii) gli intermediari che mettono in comunicazione - attraverso una piattaforma online - $i$ prestatori e utenti e che agevolano le transazioni tra di essi ("piattaforme di collaborazione")". La definizione, pertanto, comprende sia forme di scambio business to consumer (B2C), sia consumer to consumer (C2C).

Il tema centrale, di recente affrontato dalla giurisprudenza della Corte di giustizia dell'Unione europea, è se le piattaforme di intermediazione siano o meno prestatori di servizi finali e se, di conseguenza, siano tenute a essere in possesso dei requisiti (autorizzazioni o concessioni) necessari per operare in un determinato mercato. Sulla base della c.d. direttiva servizi ${ }^{18}$, i regimi di autorizzazione e i requisiti di licenza sono compatibili con il diritto dell'Unione soltanto se non discriminatori, necessari per conseguire un obiettivo di interesse generale e proporzionati rispetto a tale obiettivo. A parere della Commissione, la pressione deregolatrice creata dalla sharing economy dovrebbe indurre i legislatori nazionali a rivalutare l'efficacia e la proporzionalità della regolazione. I sistemi reputazionali generalmente implementati dalle piattaforme di intermediazione, ad esempio, potrebbero essere idonei a offrire un livello di sicurezza e tutela del consumatore, in termini di riduzione delle asimmetrie informative, pari o superiore di quello offerto da certi regimi autorizzatori. 
Per quanto riguarda lo scambio tra pari (peer to peer), gli Stati membri sono invitati a stabilire delle soglie al di sopra delle quali il privato perde il proprio stato di prestatore occasionale di servizi e diviene professionista, consentendo a coloro che si collocano al di sotto della soglia di operare senza licenze o autorizzazioni o creando regimi light ad hoc.

La questione se la piattaforma di collaborazione fornisca o meno anche i servizi sottostanti, dovrà essere risolta, invece, verificando se la stessa fissi anche il prezzo e le condizioni contrattuali tra il prestatore e l'utente, se sia proprietaria o meno dei beni utilizzati per fornire il servizio, se sia responsabile in caso di inadempimento, oppure se sussistano rapporti di lavoro subordinato tra la piattaforma e il prestatore del servizio all'utente finale.

La Comunicazione rappresenta il punto di avvio di un ampio processo di studio e di monitoraggio da parte della Commissione che, di recente, ha ricevuto una forte sponda da parte del Parlamento Europeo con una risoluzione del 15 giugno $2017^{19}$. Nel richiamare alcune criticità, in particolare sulla tutela dei diritti dei lavoratori, il Parlamento mette in evidenza come la mancanza di un quadro regolatorio chiaro e omogeneo stia impedendo al mercato europeo di veder nascere e sviluppare al suo interno piattaforme digitali, incluse quelle dell'economia collaborativa, in grado di competere con i giganti statunitensi e asiatici.

Ma natura abhorret a vacuo (la natura non tollera vuoti) e i problemi che i regolatori immaginano e auspicano di risolvere in futuro vengono portati da vecchi e nuovi operatori economici nelle aule di giustizia. Dopo una attesa non breve, la Corte di giustizia dell'Unione europea si è pronunciata su una questione pregiudiziale sollevata dal Juzgado de lo Mercantil (Tribunale delle Imprese di Barcellona) ${ }^{20}$. La vicenda contenziosa portata innanzi al giudice a quo è simile ad altre sollevate in molti paesi del mondo (compreso il Brasile ${ }^{21}$ ). Un'associazione di tassisti agiva per ottenere l'accertamento della violazione da parte di Uber delle norme in materia di trasporto pubblico non di linea ${ }^{22}$. La società statunitense offrirebbe un servizio del tutto equiparabile a quello offerto dalle auto bianche senza, però, aver previamente ottenuto una licenza, senza applicare le tariffe stabilite dal comune, senza rispettare, 
nella sostanza, il set di regole che esistono in tale mercato per assicurare la sostenibilità economica del servizio e la tutela dei consumatori.

Con riferimento al diritto dell'Unione, la questione verte sulla possibilità di inquadramento del servizio offerto da Uber tra i servizi della società dell'informazione; oppure se lo stesso debba essere qualificato come «servizio nel settore dei trasporti», ai sensi dell'articolo 58, paragrafo 1 del Trattato sul funzionamento dell'Unione europea (TFUE), e, di conseguenza, escluso dall'ambito di applicazione della disciplina della libera prestazione dei servizi (articolo 56 TFUE, direttiva 2006/123/CE) e del commercio elettronico (direttiva 2000/31/CE ${ }^{23}$ ).

La Corte ha ammesso che se il servizio si limitasse a favorire la conclusione di una transazione tra un prestatore del servizio e un utente, lo stesso si differenzierebbe dal servizio di trasporto e potrebbe rientrare nell'ambito di applicazione di diverse direttive o disposizioni del TFUE in materia di libera prestazione di servizi, costituendo un servizio della società dell'informazione. Ma, proseguendo nel proprio ragionamento, e richiamando implicitamente gli indici rivelatori indicati dalla Commissione nella citata comunicazione del 2016, la Corte ha riconosciuto che Uber organizza direttamente il servizio creando l'offerta e facendola incontrare con la domanda, fissa e riscuote le tariffe, seleziona i propri autisti, che, peraltro, possono essere esclusi in ogni momento dal servizio. L'elemento "trasporto", così come aveva evidenziato l'avvocato generale Szpunar nelle proprie conclusioni, prevale nettamente sulla componente di intermediazione del servizio, determinando che l'attività debba sottostare alla disciplina nazionale e locale in materia di trasporto pubblico non di linea, non avendo il Parlamento europeo e il Consiglio dell'Unione europea adottato norme comuni o altre misure, in base all'articolo 91, paragrafo 1, TFUE.

A conclusioni del tutto simili sono, del resto, pervenuti anche giudici italiani in alcune occasioni, pronunciandosi sul servizio Uber pop, che consente a qualsiasi utente di mettersi a disposizione per trasportare passeggeri - a differenza di Uber Black, che in Italia è operato da titolari di licenza di noleggio con conducente. Uber pop è al momento proibito sul territorio italiano per effetto di due ordinanze del Tribunale di Milano (25 maggio e 9 luglio 2015) al termine di un procedimento d'urgenza 
ex art. 700 c.p.c. In queste decisioni i giudici hanno ritenuto che Uber avrebbe posto in essere condotte anticoncorrenziali offrendo un servizio equivalente a quello dei taxi, senza rispettare la normativa nazionale, la cui compatibilità con l'ordinamento dell'Unione è stata esplicitamente valutata e trova oggi conferma nella sentenza della Corte di giustizia. Le ordinanze del Tribunale di Milano sono state seguite, con motivazione sostanzialmente analoga, e di fatto "assorbite" dalla sentenza del Tribunale di Torino del 24 marzo 2017, n. 1553.

La conformità a un quadro normativo, che definire confuso sarebbe eufemistico, del servizio Uber black è stata, invece, affermata dalla sezione specializzata in materia di impresa del Tribunale di Roma, di nuovo con ordinanza collegiale emessa a conclusione di un procedimento ex art. 700 c.p.c. Quel che rileva in questa sede è che, anche in questo caso, il Tribunale ha invocato un intervento del legislatore a fare chiarezza e a regolare il fenomeno dell'economia collaborativa (simili richieste sono anche pervenute dall'Autorità garante della concorrenza e del mercato (AGCM, segnalazioni AS 1222 del 29 settembre 2015 e AS 1354 del 10 marzo 2017) e dall'Autorità di regolazione dei trasporti (segnalazione del 21 maggio 2015).

Nel corso della precedente legislatura ci sono stati alcuni tentativi di rispondere alla richiesta di regolazione proveniente dalle corti, dalle Autorità indipendenti, dalle imprese e dagli utenti, il cui iter non è, però, giunto a conclusione ${ }^{24}$. Si segnalano, in particolare, la proposta di legge Tentori (AC 3564) alla Camera e la proposta Del Barba al Senato (AS 2268). Entrambe contenevano misure regolatorie comuni, tese a offrire un grado di minimo di garanzia dei consumatori-utenti, di tutela della privacy e di enforcement fiscale. Tutte e due le proposte prevedevano la costituzione di un albo presso l'Autorità garante della concorrenza e del mercato - ennesima manifestazione della passione nazionale per elenchi e albi, soprattutto se tenuti da Autorità indipendenti, che non cede nemmeno di fronte al più dinamico e inarrestabile processo tecnologico.

Non stupirà il fatto che, alla fine, l'unico intervento che è stato approvato è di natura tributaria. Il decreto-legge 24 aprile 2017, n. 50 ha disciplinato il regime fiscale delle locazioni brevi, prevedendo l'applicazione della c.d. cedolare secca e individuando le piattaforme 
di intermediazione come sostituti di imposta. L'AGCM, tra gli altri, ha protestato, ritenendo la norma sproporzionata; il provvedimento di attuazione adottato dall'Agenzia delle entrate è ora impugnato al Tribunale amministrativo regionale, che si deve pronunciare nel merito dopo aver respinto l'istanza cautelare.

\section{CONCLUSIONI}

Le sfide, non da oggi, rappresentate dalla digitalizzazione e dalla diffusione dell'economia collaborativa - alimentate da un quadro generale di globalizzazione dell'economia e del diritto - si presentano non solo come sfide alla regolazione o regolamentazione degli ordinamenti giuridici nazionali, ma investono talvolta le caratteristiche fondative stesse del costituzionalismo moderno e contemporaneo. Questo, nel suo nucleo, è basato sulla limitazione del potere, non solo pubblico, statuale in senso tradizionale, ma anche privato. È rispetto a quest'ultimo che nell'attualità si pongono le maggiori sfide.

Per quanto riguarda l'accesso a Internet, le principali criticità riguardano gli strumenti con i quali ampliare la platea delle persone che vi hanno accesso a condizioni tali da consentire la fruizione di servizi avanzati che si servono di connessioni Internet veloci. Nel contesto europeo e italiano, tali strumenti sono stati individuati ricercando il bilanciamento tra i principi di libera concorrenza e il perseguimento di obiettivi d'interesse generale. La realizzazione di nuove reti avviene, pertanto, nel rispetto del diritto degli aiuti di Stato e vede nel servizio universale la prospettiva ultima a cui approdare per garantire a tutti una connessione a Internet veloce, consentendo al tempo stesso lo sviluppo della concorrenza sul mercato della fornitura dei servizi digitali.

L'accesso a Internet è necessario per fruire dei servizi dell'economia collaborativa, che è solo una delle novità economiche determinate dall'evoluzione tecnologica. Certamente presenta una sfida per i regolatori. Anche in questo campo, la Commissione e il Parlamento europeo hanno richiamato al rispetto delle regole di concorrenza, stabilendo correttamente che è necessario creare il level playing 
field idoneo a rendere l'Europa non solo un mercato attraente per questi servizi, ma anche un laboratorio per la nascita e sviluppo delle piattaforme tecnologiche. La pressione alla deregolazione proveniente dall'elevata standardizzazione tecnologica, e dall'influsso di sistemi giuridici di matrice diversa da quello europeo che essa porta con sé, mette però a rischio conquiste sociali ed economiche del mercato unico che diamo, forse con troppo ottimismo, ormai per scontate: la tutela dei lavoratori, dei consumatori, della privacy, della concorrenza - solo per citarne alcune. Non possiamo che sperare in un ruolo attivo e coordinato delle istituzioni comunitarie e dei parlamenti nazionali. Consapevoli, tuttavia, che in mancanza, la regolazione della sharing economy verrà scritta, come spesso accade, dal legislatore dell'emergenza e nelle aule dei tribunali.

\section{NOTAS}

1 Il presente contributo, ancorché frutto di una riflessione comune, è da attribuirsi a Roberto Miccú quanto al paragrafo 3 e a Giacomo Roma quanto al paragrafo 2. L'introduzione e le conclusioni sono state redatte congiuntamente.

2 Il contributo riflette esclusivamente le opinioni dell'autore, senza impegnare la responsabilità dell'Istituzione di appartenenza.

3 P. DE HERT - D. KLOZA, Internet (access) as a new fundamental right. Inflating the current rights framework, in European Journal of Law and Technology, vol. 3, n. 2, 2012; S. Hick, E. F. HALPIN, E. Hoskins (a cura di), Human Rights and the Internet, New York, Palgrave Macmillan, 2000.

4 L'espressione sharing economy ha inizialmente trovato ampio risalto nella letteratura economica, per poi approdare anche a quella giuridica (v. infra). A titolo di esempio, si possono citare R. Botsman, Defining the Sharing Economy: What Is Collaborative Consumption - and What Isn't?, in FastCo.exist, 2015; C. CodAGnone - B. Mertens, Scoping the Sharing Economy: Origins, Definitions, Impact and Regulatory Issues, Joint Research Centre of the European Commission Technical Reports, Institute for Prospective Technological Studies, Digital Economy Working Paper 2016/01, 2016; I. PAIS - G. ProvASI, Sharing Economy: A Step towards the Re-Embeddedness of the Economy, in Stato e mercato, 2015, pp. 347-378; J. ScHOR, Debating the Sharing Economy, in Great Transition Initiative, 2014; ID., The Sharing Economy: Reports From Stage One, 2015; J. SCHOR - C. FitzMAURICE, Collaborating and Connecting: The Emergence of the Shating Economy, in L. REISCH - J. ThøGERSEN, Handbook of Research on Sustainable Consumption, Edward Elgar Publishing, 2015.

5 E. De Marco (a cura di), Accesso alla rete e uguaglianza digitale, Milano, Giuffrè, 2008; G. DE Minico, Accesso a Internet tra mercato e diritto sociali nell'ordinamento europeo e nazionale, in Federalismi, numero speciale n. 4, 2018; G. DE Minico (a cura di), Dalla tecnologia ai diritti. Banda larga e servizi a rete, Napoli, Jovene, 2010; P. TANZARELLA, Accesso a Internet: verso un nuovo diritto sociale?, in E. CAVASINo, G. ScAla, G. VERDE (a cura di), I diritti sociali. Dal riconoscimento alla garanzia, Napoli, ESI, 2013, pp. 517-532; M. PIETRANGELO (a cura di), Il diritto di accesso ad Internet, Napoli, ESI, 2011, pp. 59-88.

6 Dal nome dell'autorità creata da questa legge per irrogare tali sanzioni, la Haute autorité pour la diffusion des œuvres et la protection des droits sur l'Internet. 
7 Conseil constitutionnel, 10 giugno 2009, n. 2009-580-DC, Loi n² 2009-669 du 12 juin 2009 favorisant la diffusion et la protection de la création sur Internet. Su tale decisione, v. J.-M. BRUGUIÈRE, Loi «sur la protection de la création sur internet»: mais à quoi joue le Conseil constitutionnel?, in Rec. Dalloz, 2009, pp. 1770 ss.; L. MARINo, Le droit d'accès à internet, nouveau droit fondamental, in Rec. Dalloz, 2009, pp. 2045 ss.; W. BENESSIANo, L'inconstitutionnalité, sanction de l'identification d'un pouvoir de répression pénale dévalué, in Rev. fr. $d r$. const., 2010, pp. 168 ss.; nella dottrina italiana, v. G. Votano, Internet fra diritto d'autore e libertà di comunicazione: il modello francese, in Dir. informazione e informatica, 2009, pp. 533 ss.; B. CARоTTI, L'accesso alla rete e la tutela dei diritti fondamentali, in Giornale dir. amm., 2010, pp. 643 ss.; N. LuCCHI, La legge «Création et Internet»: le censure del Conseil constitutionnel, in Quaderni costituzionali, 2010, pp. 375 ss., P. PASSAGLIA, L'accesso ad Internet è un diritto (il Conseil constitutionnel francese dichiara l'incostituzionalità di parte della c.d. «legge anti file-sharing»), in Foro it., 2009, IV, pp. 473 ss.

8 Fra i primi a porre la questione della necessità di dare un fondamento costituzionale ai diritti legati a Internet va citato Stefano Rodotà (S. RоDотÀ, Una Costituzione per Internet?,in Politica del diritto, 2010, n. 3, pp. 337-352), la cui iniziativa è stata alla base anche della Dichiarazione dei diritti in Internet approvata nel 2015, il cui articolo 2 riconosce il diritto di accesso a Internet come "diritto fondamentale della persona e condizione per il suo pieno sviluppo individuale e sociale). Dello stesso autore, tra gli scritti più recenti si vedano anche Il diritto di avere diritti, Roma-Bari, Laterza, 2012; Il mondo nella rete. Quali i diritti, quali i vincoli, Roma-Bari, Laterza, 2014; Solidarietà: un'utopia necessaria, Roma-Bari, Laterza, 2014.

9 Regolamento (UE) 2015/2120 del Parlamento europeo e del Consiglio del 25 novembre 2015 che stabilisce misure riguardanti l'accesso a un'Internet aperta e che modifica la direttiva 2002/22/ CE relativa al servizio universale e ai diritti degli utenti in materia di reti e di servizi di comunicazione elettronica e il regolamento (UE) n. 531/2012 relativo al roaming sulle reti pubbliche di comunicazioni mobili all'interno dell'Unione.

10 Sui SIEG nel settore delle comunicazioni elettroniche, v. F. BASSAN, Concorrenza e regolazione nel diritto comunitario delle comunicazioni elettroniche, Torino, Giappichelli, 2002; L. BELLODI, Telecomunicazioni e concorrenza nel diritto comunitario, Napoli, ESI, 1999; D. IACovone, I servizi di pubblica utilità tra Stato, mercato, regolatore e consumatore, Bologna, il Mulino, 2014; R. PEREZ, Telecomunicazioni e concorrenza, Milano, Giuffrè, 2002.

11 A titolo non esaustivo, sul servizio universale si v. G.F. CARTEI, Il servizio universale, Milano, Giuffrè, 2002; S. Frova (a cura di), Nozione ed evoluzione del servizio universale nelle telecomunicazioni, Milano, Giuffrè, 1998; M. ClARICH, Servizio pubblico e servizio universale: evoluzione normativa e profili ricostruttivi, in Diritto pubblico, 1998, pp. 181 ss.; F. CASTELLI, Il servizio universale nelle telecomunicazioni, Milano, Franco Angeli, 1998; F. SAMPERI, Il servizio universale: concorrenza versus regolamentazione, in C. VACCA, (a cura di), regole giuridiche ed evoluzione tecnologica, Milano, EGEA, 1991, pp. 51 ss..

12 Protocollo n. 26 sui servizi di interesse generale allegato al Trattato sul funzionamento dell'Unione europea.

13 Comunicazione della Commissione sull'applicazione delle norme dell'Unione europea in materia di aiuti di Stato alla compensazione concessa per la prestazione di servizi di interesse economico generale, 2012/C 8/02, punto 48.

14 Comunicazione della Commissione "Orientamenti dell'Unione europea per l'applicazione delle norme in materia di aiuti di Stato in relazione allo sviluppo rapido di reti a banda larga", 2013/C 25/01, punto 21. Per un'analisi puntuale degli Orientamenti, v. A. KliEmann, O. SteHmann, EU State Aid Control in the Broadband Sector - The 2013 Broadband Guidelines and Recent Case Practice, in European State Aid Law Quarterly, 3/2013, pp. 493 ss.

15 Direttiva 2002/22/CE del Parlamento europeo e del Consiglio del 7 marzo 2002 relativa al servizio universale e ai diritti degli utenti in materia di reti e di servizi di comunicazione elettronica (direttiva servizio universale).

16 Nella dottrina giuridica che si è occupata del tema, v., in particolare, M. CoLANGELO - V. ZENo-ZENCovich, La intermediazione online e la disciplina della concorrenza: i servizi di viaggio, soggiorno e svago, in Diritto dell'informazione e dell'informatica, n. 1, 2015, pp. 43 ss.; P.L. PETRILLO - C. HoNORATI, Diritti e libertà alla prova dell'economia della condivisione. Prime note di confronto tra 
Europa e America Latina, in Federalismi, 2018; D. SABATo - A. LePore, Sharing economy. Profili giuridici, Napoli, ESI, 2018; G. SMORTo, Economia della condivisione e antropologia dello scambio, in Diritto pubblico comparato ed europeo, n. 1, 2017, pp. 119-140; ID., Verso la disciplina giuridica della sharing economy, n. 2, 2015, pp. 245-277

17 Comunicazione della Commissione al Parlamento europeo, al Consiglio, al Comitato economico e sociale europeo e al Comitato delle regioni, Un'agenda europea per l'economia collaborativa, COM(2016) 356 final del 2 giugno 2016.

18 Direttiva 2006/123/CE del Parlamento europeo e del Consiglio, del 12 dicembre 2006, relativa ai servizi nel mercato interno, GUUE L 376 del 27 dicembre 2006, pp. 36-68.

19 Risoluzione del Parlamento europeo del 15 giugno 2017 sulle piattaforme online e il mercato unico digitale (2016/2276(INI)).

20 Corte di giustizia dell'Unione europea, gr. sez., 20 dicembre 2017, causa C-434/15, Asociación Profesional Elite Taxi c. Uber Sustems SpainSL. In dottrina, v. O. Pollicino - V. LubELlo, Un monito complesso ed una apertura al dibattito europeo rilevante: Uber tra giudici e legislatori, in Osservatorio AIC, n. 2, 2017; A. PREviato, Il caso Uber al vaglio della Corte di giustizia europea: una sentenza che ridefinisce i termini della sharing economy, in Diritto pubblico europeo - rassegna online, 2018; G. RESTA, Uber di fronte alle corti europee, in Diritto dell'informazione e dell'informativa, n. 2, 2017, pp. 330 ss.

21 G. PACELla, Lavoro e piattaforme: una sentenza brasiliana qualifica subordinato il rapporto tra Uber e gli autisti, in Rivista italiana di diritto del lavoro, n. 3, 2017, pp. 570-578.

22 Più in generale, sul caso Uber v. A. АмAто, Uber and the Sharing Economy, in The Italian Law Journal, n. 1, 2016, pp. 171 ss.; L. BELviso, Il caso Uber negli Stati Uniti e in Europa fra mercato, tecnologia e diritto. Obsolescenza regolatoria e ruolo delle Corti, in Rivista di diritto dei media, n. 1 , 2018; A. Boitani - S. Colombo, Taxi, Ncc, Uber: scontro finale o alba di coesistenza?, in Mercato Concorrenza Regole, n. 1, 2017, pp. 61-78; E. MostACCI - A. SommA, Il caso Uber. La sharing economy nel confronto tra common law e civil law, Milano, EGEA, 2016; V. C. RomAno, Nuove tecnologie per il mitradismo regolamentare: il caso Uber Pop, in Mercato Concorrenza Regole, n. 1, 2015, pp. 133-140; D. TEgA, "Pronto Uber? Un'auto a Piazza del Quirinale n. 41": la gig economy arriva alla Corte costituzionale, in forumcostituzionale.it, 2017.

23 Direttiva 2000/31/CE del Parlamento europeo e del Consiglio dell'8 giugno 2000 relativa a taluni aspetti giuridici dei servizi della società dell'informazione, in particolare il commercio elettronico, nel mercato interno, GUUE L 178 del 17 luglio 2000, pp. 1-16.

24 G. D’IPPolito, Sharing economy: l'esperienza italiana della XVII legislatura alla luce degli orientamenti europei, in Rivista di diritto dei media, n. 2, 2018.

\section{Roberto Miccú}

Professore ordinario di Istituzioni di diritto pubblico

Sapienza - Università di Roma

\section{Giacomo Roma}

Dottorando in Diritto pubblico, comparato e internazionale curriculum Diritto pubblico dell'economia Sapienza - Università di Roma 
